\title{
The use of laser ablation technique for the synthesis of titanium dioxide nanoparticles synergistic with sulfamethoxazole to prepare an anti-corrosion surface coating for mild steel and study of refraction and absorption
}

\author{
Buraq T. SH. AL-Mosawi \\ Department of Mathematics, College of Education, Misan University, Iraq \\ E-mail: buraq@uomisan.edu.iq
}

\begin{abstract}
Titanium dioxide nanoparticles $\left(\mathrm{TiO}_{2} \mathrm{NPs}\right)$ have been prepared by the laser ablation technique. The surface structure and morphology of $\mathrm{TiO}_{2} \mathrm{NPs}$ was elucidated by ultraviolet-visible (UVVis) and Fourier transform infrared (FTIR) spectroscopies, scanning electron microscopy (SEM) and X-ray diffraction (XRD). The variable size and geometry of the nanoparticles depend on the medium used for the synthesis. Physically, titanium oxide NPs give the optimum refractive index of $1.655,1.556$ and 1.554 for the studied wavelengths $(487.1,587.3$ and $600 \mathrm{~nm}$ ), respectively. This change in refractive indices allowed this material to be a good transmittance component useful in physical and optical applications. The composition of $\mathrm{TiO}_{2}$ NPs and sulfamethoxazole was applied as a corrosion inhibitor for mild steel surface in a hydrochloric acid solution. The inhibition performance was studied by the weight loss method. The results from this study show that the new synergistic compounds act as a coating for mild steel and reduce the corrosion with an inhibition efficiency of 93.6\%. The Langmuir adsorption isotherm model was utilized to evaluate the adsorption equilibrium of the corrosion inhibitor.
\end{abstract}

Keywords: $\mathrm{TiO}_{2} \mathrm{NPs}$, mild steel, laser ablation, sulfamethoxazole, corrosion inhibitor.

Received: July 2, 2020. Published: August 19, 2020

doi: $\underline{10.17675 / 2305-6894-2020-9-3-15}$

\section{Introduction}

There is a great interest of researchers in the field of nanotechnology in preparing and using $\mathrm{TiO}_{2}$ nanoparticles because they have a considerable specific area [1], and yet they suffer from a lack of recycling in aqueous liquids. $\mathrm{TiO}_{2}$ nucleic particles were prepared using chemical and physical techniques, for example, chemical precipitation (CBD) [2], electronic beam evaporation [3], interactive electronic beam evaporation [4], spray magnetron [5], solution gel [6], and thermal oxidation [7]. Titanium dioxide NPs exhibited a significant decrease in the band gap $(3.2 \mathrm{eV})$ in addition to an improvement in the optical characteristics, thereby improving their photocatalytic efficiency in visible light. The sunlight is preferred as it is economical and environment friendly. Many theoretical calculations have been made to illustrate the effect of $\mathrm{TiO}_{2}$ anion stereochemical properties on band gap adjustments [8]. However, the synergistic effect of inhibiting mild steel corrosion when an inorganic 
compound is added to an acidic solution that contains an organic compound is very important as the synergistic effect increases the inhibition efficiency. Some mercaptotriazole derivatives with different heteroatoms and substances were synthesized. The findings of this researcher revealed that all heteroatoms favored a high quality of corrosion inhibitor for mild steel in $\mathrm{HCl}$ solution [9]. The researchers also reported that some $\mathrm{N}$ - and S-containing heterocyclic compounds are eco-friendly inhibitors compared to commercial inhibitors that may be toxic, like nitrite. Recently, studies on the use of synergistic effects in corrosion inhibitors intensified because of there are many adsorption centers that facilitate the formation of complexes with metal ions. The complexes that are formed are adsorbed on a metal surface to create a film (or barrier film) to separate the metal surface from the corrosive media [10]. The released energy allows the researcher to determine the inhibition effectiveness. Organic molecules containing polar functional groups, for example, nitrogen, sulfur, as well as oxygen in a conjugated system proved to be successful as corrosion inhibitors for steel [11]. Some of such organic compounds have multiple active centers. In some reports, the inhibitive power of these compounds is related to the structure of the rings and heteroatoms which are significant active centers of adsorption [12]. The term corrosion refers to the material (metal such as steel) destruction by chemical or electrochemical interaction with the surrounded medium [13]. Corrosion can ruinously harm the metal and compound structures causing monetary results such as repair, replacement, item loss and contamination of products. Because of these unsafe impacts, corrosion is a bothersome phenomenon that should be inhibited. Several methodologies such as inhibitors are applied to reduce the corrosion rates and the rates at which an inhibitor can spread with a perspective on improving the metal lifetime and compound materials [14]. The corrosion inhibitors are compounds that reduce the corrosion rate when added in a small amount to a specific medium. Two classes of such inhibitors are categorized: (a) The first one is that depends on an oxidizing agent, so it creates a protective oxide layer and (b) The second one is that works selectively to prevent corrosion by absorption, as it creates a barrier to the arrival of surfactants. Most of the heteroatom-containing molecules are costly and possess high toxicity. Consequently, these drawbacks provoked the quest for their replacement [15].

The aim of this work is to prepare titanium dioxide nanoparticles $\left(\mathrm{TiO}_{2} \mathrm{NPs}\right)$ by laser ablation technique. The surface structure and morphology of $\mathrm{TiO}_{2} \mathrm{NPs}$ was elucidated by ultraviolet-visible (UV-Vis) and Fourier transform infrared (FTIR) spectroscopies, scanning electron microscopy (SEM) and X-ray diffraction (XRD). The synergistic combination of $\mathrm{TiO}_{2}$ NPs and sulfamethoxazole was applied as a corrosion inhibitor for mild steel surface in hydrochloric acid solution. The inhibition performance was studied by weight loss method. Langmuir adsorption isotherm model was utilized to evaluate the adsorption equilibrium of the corrosion inhibitor. 


\section{Experimental}

\subsection{Synthesis of $\mathrm{TiO}_{2}$ nanoparticles}

The preparation of titanium oxide by pulsed laser ablation (PLA) was conducted in double distilled water. A titanium sample immersed in a water solution was exposed to an Nd:YAG pulsed laser that was focused by a lens. A power meter was utilized to evaluate the energy of the focused laser. The ablated titanium was oxidized directly and reacted with water molecules to give $\mathrm{TiO}_{2}$ NPs. The morphology of the ablated titanium surface was investigated by a scanning electron microscope (SEM). UV-Visible spectroscopy was used to determine the absorption spectrum.

\subsection{Corrosion application}

The standard of this technique is based on the experimental determination of the weight loss of a specimen surface during the period of exposure in an acidic medium, without and with addition of the tested inhibitor (synergistic with $\mathrm{TiO}_{2} \mathrm{NPs}$ ), at a solution temperature of $303 \mathrm{~K}$. The weight loss examinations were conducted in the aerated medium. The hydrochloric acid solution volume was $100 \mathrm{~mL}$, and the specimens had rectangular shapes with dimensions of $2.5 \mathrm{~cm} \times 4 \mathrm{~cm} \times 0.2 \mathrm{~cm}$. Specimens were polished and washed with double distilled water, then acetone, and dried in a desiccator. The specimens were accurately weighted and exposed to the acidic medium in the absence and in the presence of various inhibitor (and $\mathrm{TiO}_{2} \mathrm{NPs}$ ) concentrations at temperatures of $303,313,323$ and $333 \mathrm{~K}$ for 10 hours exposure time.

\section{Results and Discussion}

\subsection{Synthesis of $\mathrm{TiO}_{2} \mathrm{NPs}$}
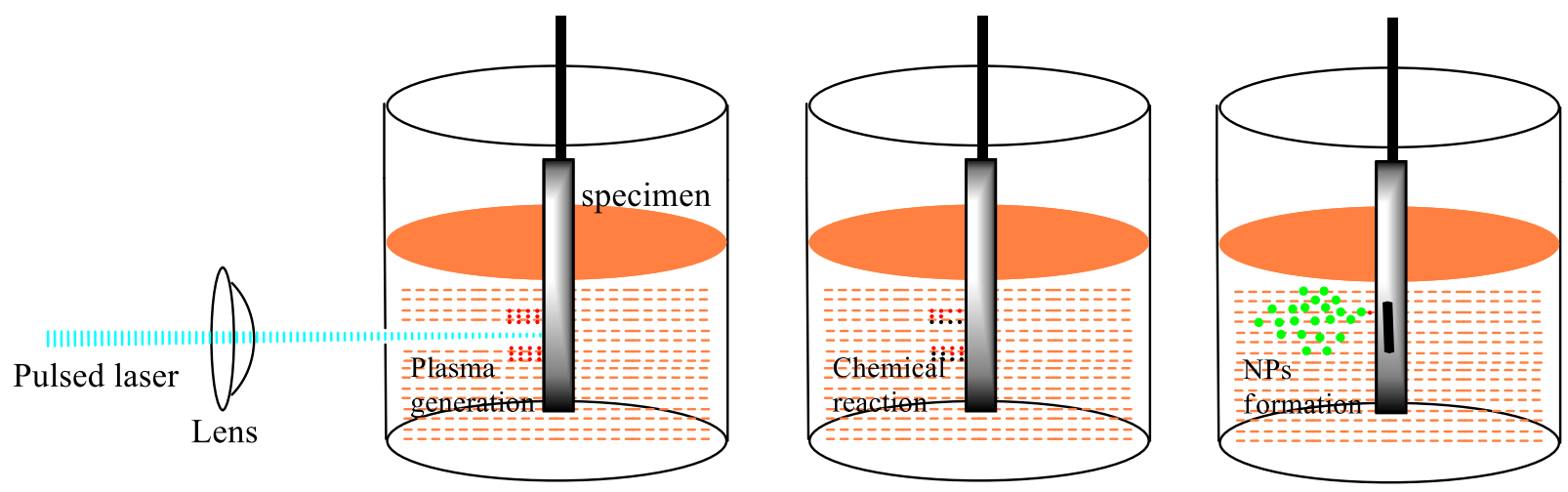

Figure 1. $\mathrm{TiO}_{2}$ NPs formation by PLA in solution.

Pulsed laser ablation (PLA) in solutions is a significant chemical-physical technique for obtaining nanoparticles (NPs) in liquids. First of all in this technique, irradiation of pulsed high energy laser induces chemical and physical processes which lead to the production of metal NPs. The ablation technique is schematically shown in Figure 1. The plasma cloud is 
formed firstly, then radiation-plasma interaction occurs, followed by a chemical process and finally formation of metal NPs in solution. The interactions between the solution and the plasma formed shock waves with high energy in the liquid, which induced chemical reactions between the specimen, particles and solution. The particles which were generated remain in solution and participate in the reaction.

\section{2. $\mathrm{TiO}_{2}$ nanoparticles characterization}

The structure of $\mathrm{TiO}_{2}$ NPs was characterized by UV-Vis spectroscopy, scanning electron microscopy and X-ray diffraction.

\subsubsection{Ultraviolet-visible absorption spectroscopy}

$\mathrm{UV}$-Vis spectroscopy is an efficient technique to investigate the properties of $\mathrm{TiO}_{2}$ nanoparticles directly in the reaction solution that allow the determination of some properties such as the energy gap. UV-Vis absorbance is a powerful method to see and explain the strong absorption of UV light $(<400 \mathrm{~nm})$ shown by $\mathrm{TiO}_{2} \mathrm{NPs}$ samples. UV-Vis transmittance gives a way to determine the light transmittance through the samples and to determine the refractive index in the visible region. The two spectroscopy patterns were obtained by a Refurbished Shimadzu Pharma-Spec UV-1700 Double Beam Scanning UVVis Spectrophotometer. From these results, and also from Figure 2, the light transmittance exceeded 0.8 and 0.9 in some regions of the visible spectrum. Still, the sample is transparent to the visible light. This means that the synthesized $\mathrm{TiO}_{2} \mathrm{NPs}$ can be utilized as a material for photocatalytic applications.

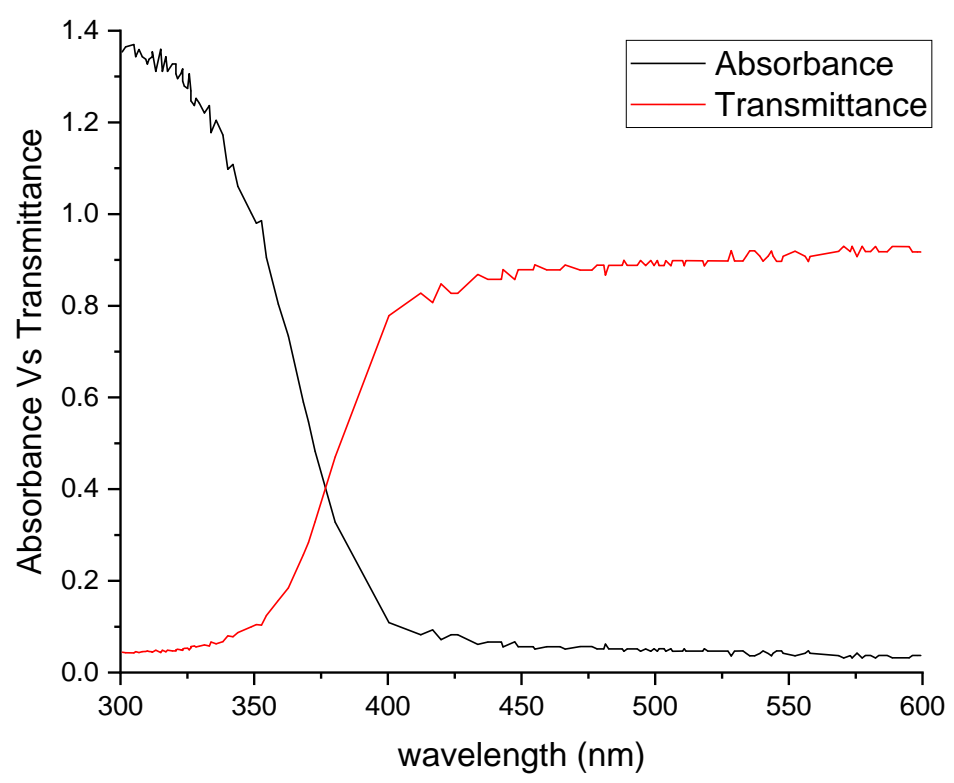

Figure 2. Absorbance and transmittance of $\mathrm{TiO}_{2}$ nanoparticles versus the wave length. 


\subsubsection{Refractive index variation with wavelength}

The refractive index of the prepared nanocomposites increased on increasing the sample density by adding $\mathrm{TiO}_{2} \mathrm{NPs}$ according to the formula $n=c / v$, where $c=3 \cdot 10^{8} \mathrm{~m} / \mathrm{s}$. The degradation in refractive index values toward the red shift is expected because the refractive index is a function of wavelength. From Figure 3, the variation in refractive indices at three points of $487.1,587.3$ and $600 \mathrm{~nm}$ was observed. The obtained refractive indices at the mentioned points are $1.655,1.556$ and 1.554 , respectively. This variation is ascribed to the dispersion phenomenon that affects the sample particles (Figure 3).

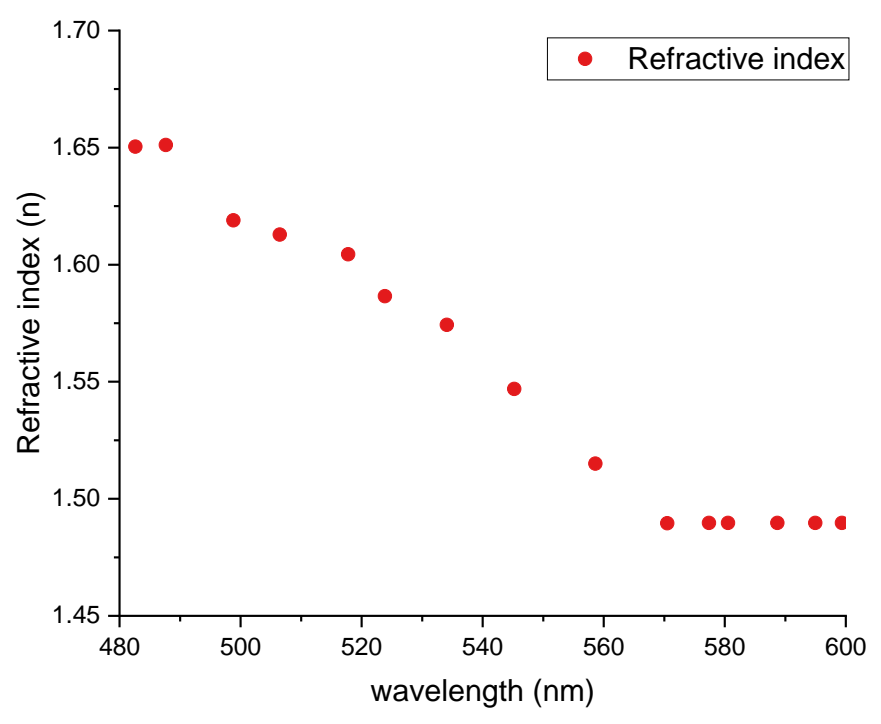

Figure 3. Refractive index of $\mathrm{TiO}_{2}$ nanoparticles versus wavelength in $\mathrm{nm}$.

\subsubsection{Scanning electron microscopy}

The scanning electron microscope image of titanium oxide NPs which were synthesized by pulse laser ablation technique shown in Figure 4 exhibit that there are irregular shapes of $\mathrm{TiO}_{2}$ nanoparticles, imputed to the pulse laser ablation and the reaction of the species with the experimental solution. Titanium oxide NPs are presented in Figure 4. The average sizes of $\mathrm{TiO}_{2}$ NPs were $70 \mathrm{~nm}$.

\subsubsection{X-Ray}

The initial characterization of crystallite size variation was conducted by Scherrer in 1918 [16]. However the initial accurate opinion of line broadening was reported in 1944. Williamson size-strain theory was progressing in 1953 as a technique to separate size and strain impacts through angles $[17,18]$. The diffraction peaks of $\mathrm{TiO}_{2}$ found at $2 \theta=26.04^{\circ}$, $42.95^{\circ}$ and $57.03^{\circ}$ were assigned to (100), (101) and (111) planes. The diffraction peaks at these angles indicated that $\mathrm{TiO}_{2}$ was in anatase phase. XRD peak intensity of the sample means that the NPs were crystalline while the broad peaks indicated small crystallite size. 


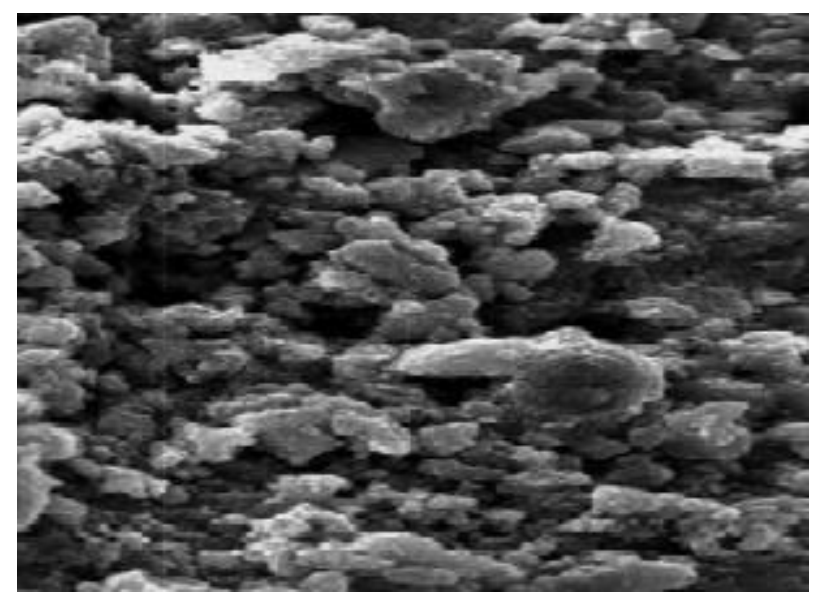

Figure 4. SEM micrograph of $\mathrm{TiO}_{2}$ nanoparticles.

There was a slight shift in $2 \theta$ values that confirmed that the NPs positioned at the higher angles have a slightly larger particle size than that at lower angles. The crystallite size of $\mathrm{TiO}_{2}$ NPs was calculated using Scherrer equation:

$$
D=\frac{k \lambda}{\beta \cos \theta},
$$

where $k=0.9$ is a dimensionless geometric factor accounting for the particle shape, $\lambda$ is the $\mathrm{X}$-ray wavelength, $\beta$ is the full width at half maximum (FWHM) of the most intense diffraction peak and $\theta$ is the diffraction angle. The average particle size of synthesized NPs was found to be $10 \mathrm{~nm}$ according to the XRD data.

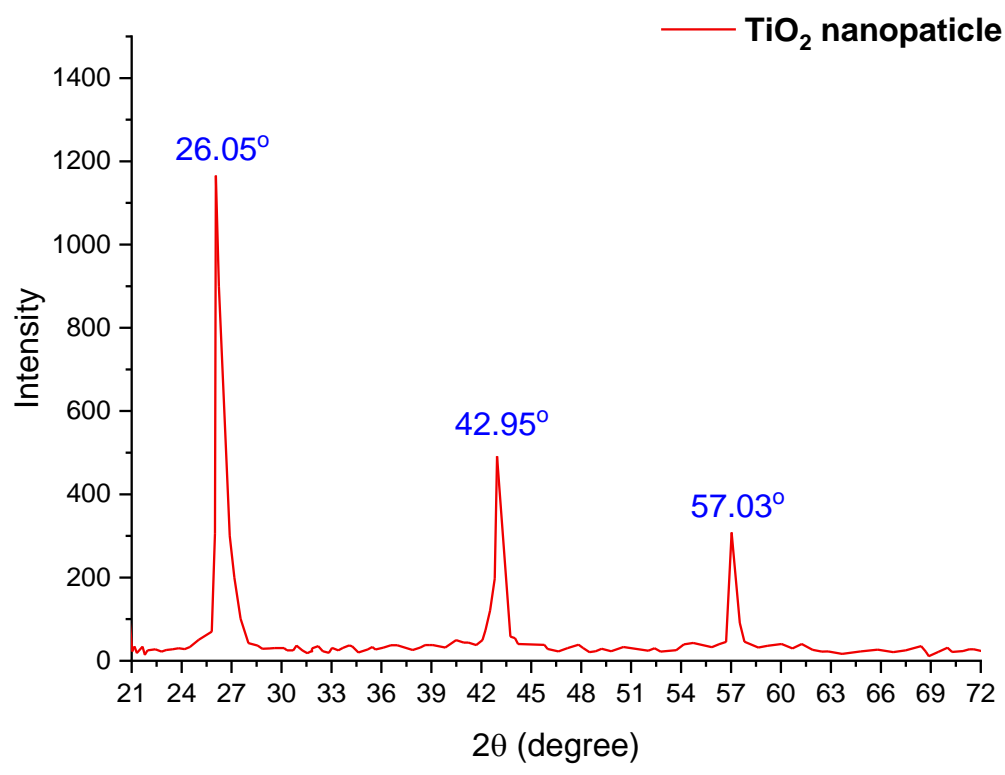

Figure 5. XRD of $\mathrm{TiO}_{2}$ nanoparticles. 


\subsection{Application as a corrosion inhibitor}

\subsubsection{Weight loss method}

The chemical structure of the tested inhibitor is presented in Figure 6. The concentration effect is evaluated by the immersion of specimen in the hydrochloric acid medium, in the absence and in the presence of the tested inhibitor sulfamethoxazole at various concentrations $(50,100,200$ and $500 \mathrm{mg} / \mathrm{L})$. The corrosion inhibition efficiency was evaluated after immersion for $10 \mathrm{~h}$ at $303 \mathrm{~K}$. The corrosion rate and the inhibition efficiency were calculated by Equations (1) and (2).

$$
\begin{gathered}
W=\frac{W_{\mathrm{b}}-W_{\mathrm{a}}}{A t} \\
\operatorname{IE}(\%)=\frac{W-W_{\mathrm{inh}}}{W},
\end{gathered}
$$

where $W_{\mathrm{b}}$ is the initial weight of tested specimen, $W_{\mathrm{a}}$ is the weight of specimen after immersion in the acid, $W$ is the corrosion rate in the absence of sulfamethoxazole and $W_{\text {inh }}$ is the corrosion rate in the presence of sulfamethoxazole. $A$ is the specimen surface area $\left(\mathrm{cm}^{2}\right)$ and $t$ is the immersion time (h). The corrosion rate values and the inhibitive efficiency $(I E \%)$ determined by gravimetric measurements at various inhibitor concentrations are presented in Figure 7.

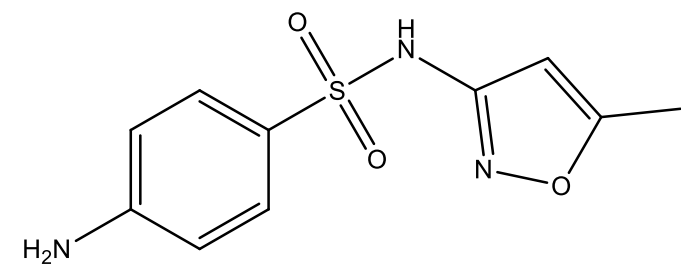

Figure 6. Chemical structure of sulfamethoxazole.

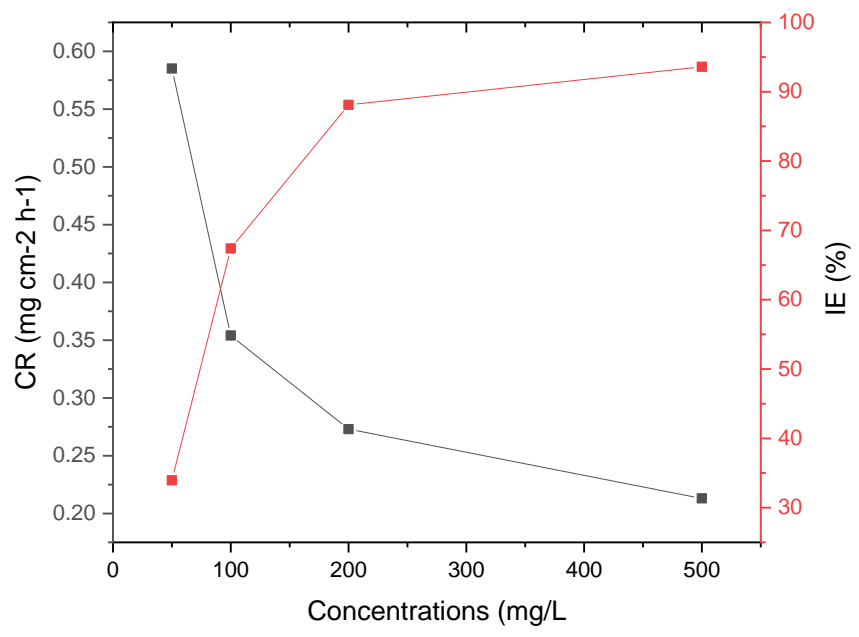

Figure 7. Inhibition efficiency and corrosion rate of the metal in acidic solution with various concentrations of sulfamethoxazole. 


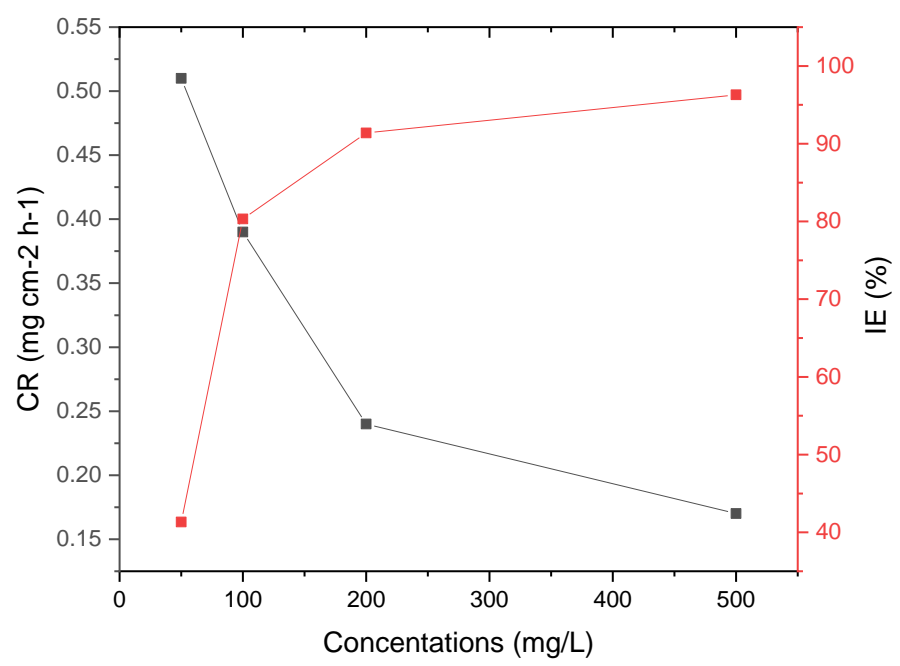

Figure 8. Inhibition efficiency and corrosion rate of the metal in acidic solution with various concentrations of sulfamethoxazole and $0.001 \mathrm{mg}$ of $\mathrm{TiO}_{2}$.

The corrosion rate values and the inhibitive efficiency (IE\%) determined by gravimetric measurements at various inhibitor concentrations in the presence of $\mathrm{TiO}_{2} \mathrm{NPs}(0.01 \mathrm{mg})$ are presented in Figure 8.

The results from Figures 7 and 8 exhibit that the corrosion rate decreases with an increase in the concentration of sulfamethoxazole and decreases even more upon addition of $\mathrm{TiO}_{2}$ NPs. The inhibition efficiency increases with addition of sulfamethoxazole to become 93\% at the highest tested inhibitor concentration. Moreover, the addition of $\mathrm{TiO}_{2} \mathrm{NPs}$ $(0.01 \mathrm{mg})$ to the corrosive solution increases the inhibition efficiency at the same sulfamethoxazole concentration.

\subsubsection{Adsorption isotherm}

The surface coverage $(\theta)$ values at various sulfamethoxazole concentrations as determined by the weight loss method were utilized to evaluate the adsorption isotherm of sulfamethoxazole in the corrosive medium on the tested mild steel. The surface coverage was evaluated according to Equation 3.

$$
\theta=\frac{I E}{100}
$$

From Figure 9 one can see that the adsorption process obeys the Langmuir isotherm. From Equation 4, we can calculate the Langmuir isotherm parameters.

$$
\frac{C_{\text {inh }}}{\theta}=\frac{1}{K_{\text {ads }}+C_{\text {inh }}},
$$

where $K_{\text {ads }}$ represents the equilibrium constant, $C_{\mathrm{inh}} / \theta$ is the ratio which determines the adsorption isotherm. 


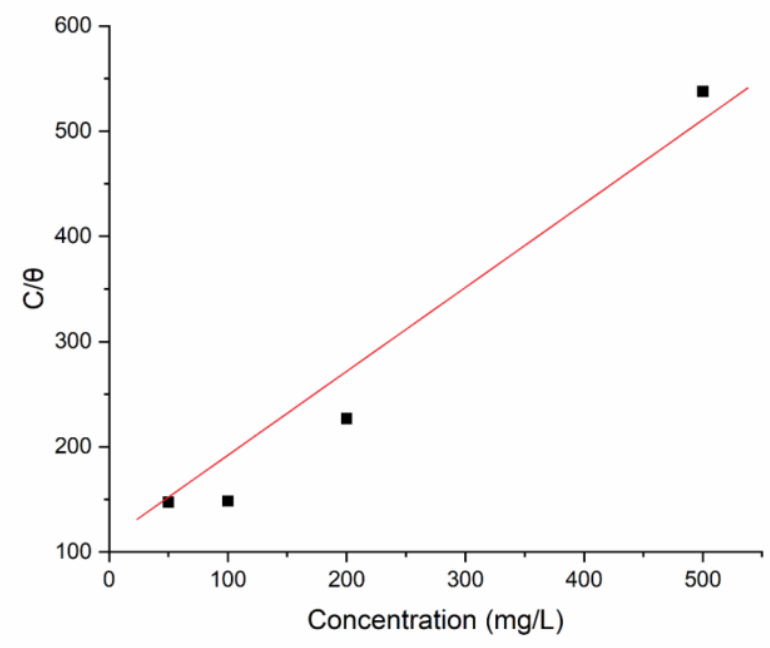

Figure 9. Langmuir adsorption of sulfamethoxazole on mild steel surface in acidic medium at $303 \mathrm{~K}$.

The Arrhenius equation gives the relation between corrosion rate, temperature and activation energy as presented in Equation 5.

$$
C R=A \exp \left(\frac{E_{\mathrm{a}}}{R T}\right)
$$

Figure 10 represents the plot of the corrosion rate versus 1/T determined from gravimetric measurmentss in the acidic medium with addition of sulfamethoxazole inhibitor at a concentration of $500 \mathrm{mg} / \mathrm{L}$ which is a straight line.

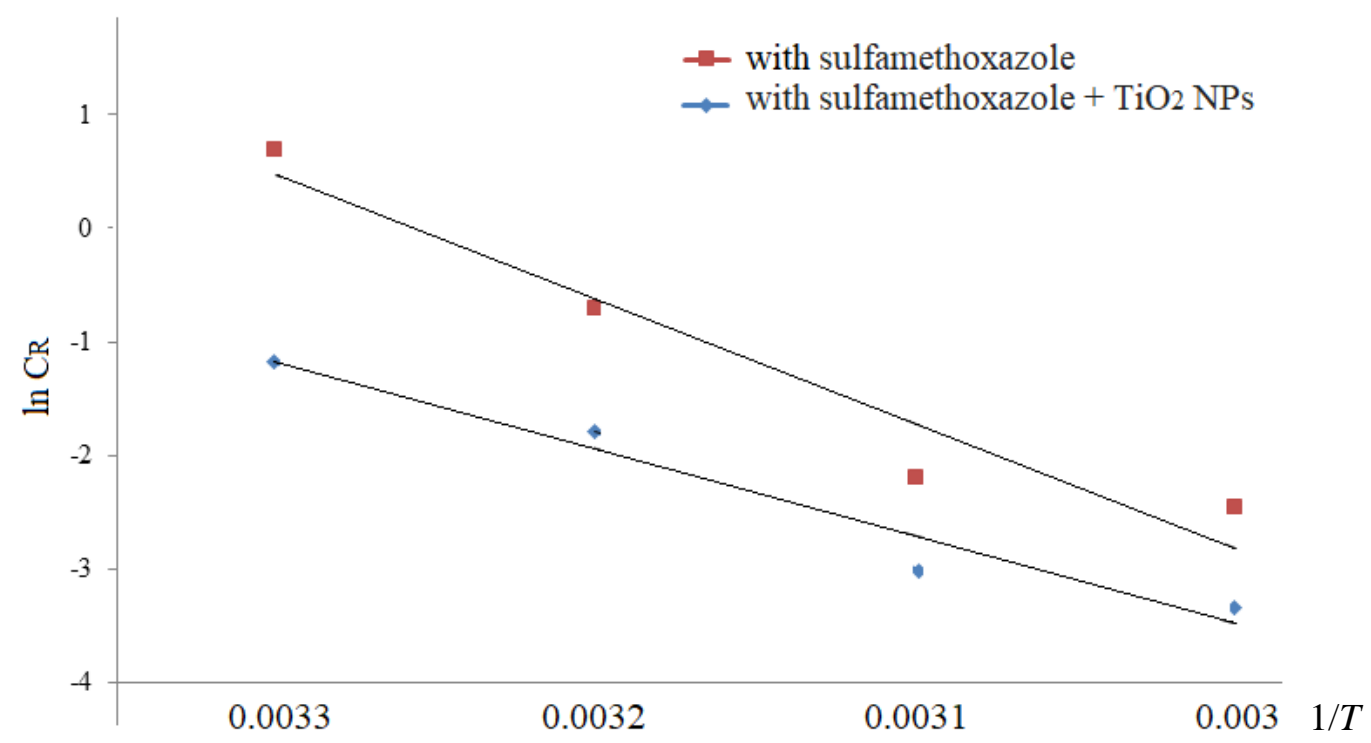

Figure 10. Arrhenius plot in acidic medium for mild steel with sulfamethoxazole in the absence and in the presence of $\mathrm{TiO}_{2} \mathrm{NPs}$. 
From Table 1, the activation energy was evaluated depending on the slope which is equal to $-E_{\mathrm{a}} / 2.303 R$.

Table 1. Physical factors of steel in acidic medium with sulfamethoxazole in the absence and in the presence of $\mathrm{TiO}_{2} \mathrm{NPs}$ at the temperatures studied.

\begin{tabular}{cc}
\hline Solution of $\mathbf{5 0 0} \mathbf{~ m g} / \mathbf{L}$ of sulfamethoxazole & $\boldsymbol{E}_{\mathbf{a}}(\mathbf{k J} / \mathbf{m o l})$ \\
\hline Absence of $\mathrm{TiO}_{2}$ & 51.47 \\
Presence of $\mathrm{TiO}_{2}$ & 58.83 \\
\hline
\end{tabular}

\subsubsection{SEM}

The surface morphology study was conducted utilizing the surface of the metal immersed in hydrochloric acid medium without and with addition of sulfamethoxazole at the highest tested concentration. The micrograph of the metal surface in the absence of sulfamethoxazole has cracks as in Figure 11-1, while in the presence of sulfamethoxazole as a corrosion inhibitor the micrograph of the surface of the tested metal was smooth. It was attributed to the formation of layer of sulfamethoxazole molecules adsorbed on the mild steel surface as shown in Figure 11-2.

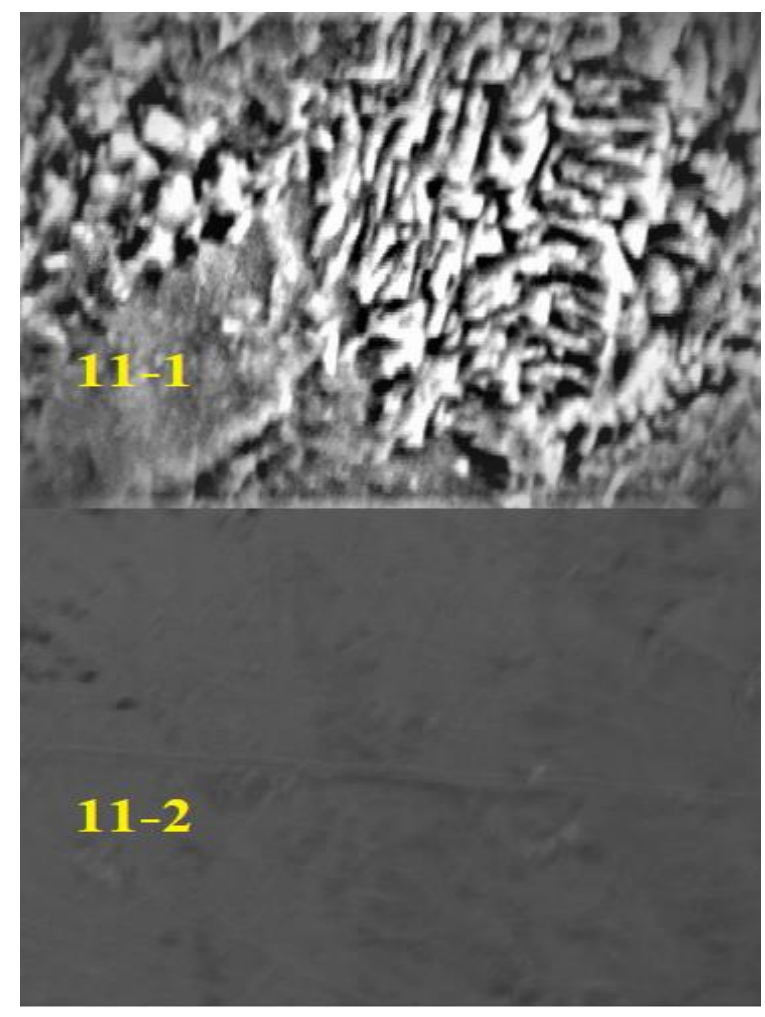

Figure 10. The SEM images of mild steel surface without (10-1) and with (10-2) sulfamethoxazole in $1 \mathrm{M} \mathrm{HCl}$ solution. 


\section{Conclusion}

$\mathrm{TiO}_{2}$ was synthesized by laser ablation technique. The XRD and SEM techniques suggest that the size of $\mathrm{TiO}_{2}$ nanoparticles was less than $100 \mathrm{~nm}$. Sulfamethoxazole in the presence and in the absence of $\mathrm{TiO}_{2}$ NPs was applied as a corrosion inhibitor for mild steel in hydrochloric acid solution. The inhibition efficiency increases with an increase in the concentration of sulfamethoxazole. The addition of $\mathrm{TiO}_{2}$ to the corrosive solution increases the inhibition efficiency from 93 to $96 \%$. The adsorption of sulfamethoxazole in the presence and in the absence of $\mathrm{TiO}_{2}$ NPs on mild steel in the corrosive medium follows the Langmuir adsorption isotherm. The images from the SEM studies show the formation of a protective layer on the metal surface.

\section{Conflict of interests}

The authors declare that there is no conflict of interests regarding the publication of this paper.

\section{References}

1. M. Farbod and M. Khademalrasool, Synthesis of $\mathrm{TiO}_{2}$ nanoparticles by a combined solgel ball milling method and investigation of nanoparticle size effect on their photocatalytic activities, Powder Technol., 2011, 214, 344-348. doi: 10.1016/j.powtec.2011.08.026

2. H. Zhu, J. Yang, S. Feng, M. Liu, J. Zhang and G. Li, Growth of $\mathrm{TiO}_{2}$ nanosheet-array thin films by quick chemical bath deposition for dye-sensitized solar cells, Appl. Phys. A, 2011, 105, 769-774. doi: 10.1007/s00339-011-6513-y

3. J.-K. Yao, H.-Y. Li, Z.-X. Fan, Y.-X. Tang, Y.-X. Jin, Y.Zhao, H.-B. He and J.D. Shao, Comparison of $\mathrm{TiO}_{2}$ and $\mathrm{ZrO}_{2}$ films deposited by electron-beam evaporation and by sol-gel process, Chin. Phys. Lett., 2007, 24, no. 7, 1964-1966. doi: 10.1088/0256-307X/24/7/049

4. Ö. Duyar, F. Placido and H. Zafer Durusoy, Optimization of $\mathrm{TiO}_{2}$ films prepared by reactive electron beam evaporation of $\mathrm{Ti}_{3} \mathrm{O}_{5}$, J. Phys. D: Appl. Phys., 2008, 41, no. 9, Article ID 095307. doi: 10.1088/0022-3727/41/9/095307

5. B. Cojocaru, Ş. Neaţu, E. Sacaliuc-Pârvulescu, F. Lévy, V.I. Pârvulescu and H. Garcia, Influence of gold particle size on the photocatalytic activity for acetone oxidation of $\mathrm{Au} / \mathrm{TiO}_{2}$ catalysts prepared by dc-magnetron sputtering, Appl. Catal., B, 2011, 107, 140-149. doi: $10.1016 /$ j.apcatb.2011.07.007

6. Z. Luo, H. Cai, X. Ren, J. Liu, W. Hong and P. Zhang, Hydrophilicity of titanium oxide coatings with the addition of silica, Mater. Sci. Eng., B, 2007, 138, no. 2, 151-156. doi: 10.1016/j.mseb.2006.06.019

7. J. Zhang, C. Pan, P. Fang, J. Wei and R. Xiong, Mo $+\mathrm{C}$ codoped $\mathrm{TiO}_{2}$ using thermal oxidation for enhancing photocatalytic activity, ACS Appl. Mater. Interfaces, 2010, 2, no. 4, 1173-1176. doi: $\underline{10.1021 / \mathrm{am} 100011 \mathrm{c}}$ 
8. I. Ahamad and M.A. Quraishi, Bis (benzimidazol-2-yl) disulphide: an efficient water soluble inhibitor for corrosion of mild steel in acid media, Corros. Sci., 2009, 51, no. 9, 2006-2013. doi: 10.1016/j.corsci.2009.05.026

9. Q.B. Zhang and Y.X. Hua, Corrosion inhibition of mild steel by alkylimidazolium ionic liquids in hydrochloric acid, Electrochim. Acta, 2009, 54, no. 6, 1881-1887. doi: 10.1016/j.electacta.2008.10.025

10. W. Li, Q. He, C. Pei and B. Hou, Experimental and theoretical investigation of the adsorption behaviour of new triazole derivatives as inhibitors for mild steel corrosion in acid media, Electrochim. Acta, 2007, 52, no. 22, 6386-6394. doi: 10.1016/j.electacta.2007.04.077

11. R. Solmaz, G. Kardas, B. Yazicı and M. Erbil, Inhibition effect of rhodanine for corrosion of mild steel in hydrochloric acid solution, Prot. Met., 2005, 41, no. 6, 581585. doi: $10.1007 / \mathrm{s} 11124-005-0083-3$

12. G. Kardas, The inhibition effect of 2-thiobarbituric acid on the corrosion performance of mild steel in $\mathrm{HCl}$ solution, Fiz.-Khim. Mekh. Mater., 2005, 41, no. 3, 337-343.

13. M. Yadav, D. Behera and U. Sharma, Development of corrosion inhibitors used in acidization of petroleum oil well, Chem. Sin., 2012, 3, 262-268.

14. M. Yadav, D. Behera and U. Sharma, Nontoxic corrosion inhibitors for N80 steel in hydrochloric acid, Arabian J. Chem., 2016, 9, S1487-S1495. doi: 10.1016/j.arabjc.2012.03.011

15. A.R. Stokes and A.J.C. Wilson, The diffraction of $\mathrm{X}$ rays by distorted crystal aggregates - I, Proc. Phys. Soc., 1944, 56, 174. doi: 10.1088/0959-5309/56/3/303

16. P. Scherrer, Nachrichten von der Gesellschaft der Wissenschaften zu Göttingen, 1918, 98-100.

17. K. Thamaphat, P. Limsuwan and B. Ngotawornchai, Phase Characterization of $\mathrm{TiO}_{2}$ Powder by XRD and TEM, Kasetsart J.: Nat. Sci., 2008, 42, no. 5, 357-361.

18. S. El-Sherbiny, F. Morsy, M. Samir and O.A. Fouad, Synthesis, Characterization and Application of $\mathrm{TiO}_{2}$ Nanopowders as Special Paper Coating Pigment, Appl. Nanosci., 2014, 4, 305-313. doi: 10.1007/s13204-013-0196-y 